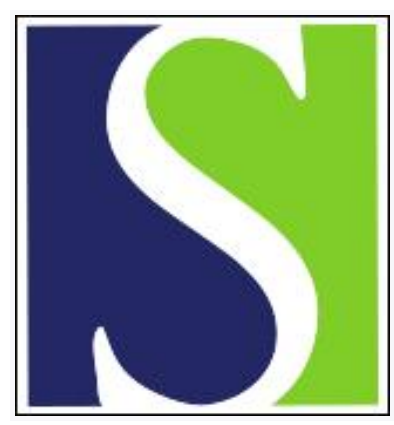

Scand J Work Environ Health 2007;33(3):215-222

https://doi.org/10.5271/sjweh.1130

Issue date: 30 Jun 2007

Prediction of cardiocerebrovascular and other significant disease from disturbed sleep and work strain

by Leineweber $\mathrm{C}$, Kecklund $\mathrm{G}$, Orth-Gomér $\mathrm{K}$

Affiliation: Karolinska Institutet, Department of Public Health Sciences, PO Box 220, 17177 Stockholm, Sweden. constanze.leineweber@ki.se

Key terms: cardiocerebrovascular disease; coronary disease; disturbed sleep; job strain; prediction; sick leave; woman; work strain

This article in PubMed: www.ncbi.nlm.nih.gov/pubmed/17572831

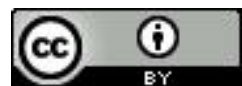




\title{
Prediction of cardiocerebrovascular and other significant disease from disturbed sleep and work strain
}

\author{
by Constanze Leineweber, PhD, ${ }^{1}$ Göran Kecklund, PhD, ${ }^{1,2}$ Kristina Orth-Gomér, PhD ${ }^{1}$
}

\begin{abstract}
Leineweber C, Kecklund G, Orth-Gomér K. Prediction of cardiocerebrovascular and other significant disease from disturbed sleep and work strain. Scand J Work Environ Health 2007;33(3):215-222.
\end{abstract}

\begin{abstract}
Objectives This study aimed at describing women's sleeping habits over a period of 9 years and at examining sleep disturbances in relation to cardiovascular, cerebrovascular, metabolic and other diseases, employment status, and work strain.

Methods Altogether 300 healthy women aged 30 to 65 years, randomly selected from the normal population, completed questionnaires at baseline about their sleep, work strain, and lifestyle and were examined for cardiovascular risk factors. The participants were then followed for 9 years and reexamined. The women who contracted a cardiovascular, cerebrovascular, or metabolic disease $(\mathrm{N}=27)$ were compared with those who developed other diseases $(\mathrm{N}=123)$ and those who remained healthy $(\mathrm{N}=140)$ during the follow-up period.

Results The women with a cardiovascular, cerebrovascular, or metabolic disease in the follow-up examination had higher cholesterol levels $(\mathrm{P}<0.001)$, a more sedentary lifestyle $(\mathrm{P}<0.01)$, and more work strain $(\mathrm{P}=0.02)$ at baseline than those who remained healthy. They also had more sleep disturbances with a borderline significant difference at baseline $(\mathrm{P}=0.07)$. In the follow-up examination both the women with a cardiovascular, cerebrovascular, or metabolic disease and those with other illnesses more frequently reported disturbed sleep than those who remained healthy $(\mathrm{P}=0.002)$. Poor sleep predicted the incidence of illness. Work strain was associated with disturbed sleep at baseline $(\mathrm{P}<0.001)$ but not at the end of the follow-up.

Conclusions General and cardiovascular health are important factors for middle-aged women's subjectively rated sleep quality, and poor sleep can, to some degree, be an early sign of subclinical cardiocerebrovascular disease. Work strain affects sleep in the acute phase but seems to have no long-term effects on sleep quality.
\end{abstract}

Key terms coronary disease; disturbed sleep; job strain; sick leave; woman.

Insomnia, characterized by difficulties maintaining or obtaining good sleep quality, is a significant health concern worldwide. Swedish studies have found a prevalence of $13-21 \%$ for sleep difficulties among men and a prevalence of $24-31 \%$ among women (1-3). Epidemiologic studies providing data on insomnia diagnoses are scarce, the prevalence being around $6-10 \%(4,5)$. However, comparisons of prevalence are difficult to interpret as prevalence depends on the definition of insomnia, the population examined, and the survey method.

Poor sleep has a strong relation to mortality and ill health. Wingard \& Berkman (6) found that women reporting sleep that was too long ( $\geq 9$ hours) or too short ( $\leq 6$ hours) had a $60 \%$ increase in mortality risk. Kripke et al $(7,8)$ found that people sleeping $7-8$ hours had higher survival rates than those sleeping less or more.
Not only an increased all-cause mortality risk, but also specific disease risks, such as cardiovascular risk, have been found to be related to severe sleep disturbances, even after control for coronary risk factors (9-11). In the Framingham study (11), a more than threefold risk of acute myocardial infarction was found for women reporting trouble falling asleep. Other studies of insomnia and coronary events have shown a 50-200\% increased risk of coronary heart disease for people with sleep problems. As we have shown in an earlier paper, poor sleep quality more than doubles the risk for a recurrent event among female cardiac patients after adjustment for cardiovascular risk factors (12).

Sleep is usually closely related to stress $(13,14)$, in particular, stress at work. However, generally the effects of shift work or occupational status (blue- versus

1 Karolinska Institutet, Department of Public Health Sciences, Stockholm, Sweden.

2 National Institute for Psychosocial Medicine, Stockholm, Sweden.

Reprint requests to: Dr C Leineweber, Karolinska Institutet, Department of Public Health Sciences, PO Box 220,17177 Stockholm, Sweden. [E-mail: constanze.leineweber@ki.se] 
white-collar work) have been investigated. Fewer studies have examined the effects of work strain on sleep quality. Furthermore, although women report more sleep problems, most of the studies investigating the "work-sleep" relationship have been carried out among men.

Using a longitudinal design, we examined a group of healthy women, representative of the female population of Stockholm, aged 30-65 years. We assessed their cardiovascular standard risk factors, sleep and work strain, as measured at baseline, in relation to diagnosed illness and cardiocerebrovascular health outcome (including diabetes) after a period of 9 years. Furthermore, we hypothesized that disturbed sleep would predict cardiocerebrovascular events during the 9 -year period. Finally, we set illness, cardiocerebrovascular health, labor market status (ie, employed, chronically ill, retired), both measured at the follow-up, and work strain (measured at baseline) in relation to sleep measured both at baseline and in the follow-up examination.

\section{Study population and methods}

\section{Study population}

The study design, recruitment, and examination procedures have been previously described elsewhere in detail (15). In this article we have focused on characteristics of a healthy control group (part of the Stockholm Female Coronary Risk Study), which consisted of 300 healthy women between 30 and 65 years of age and randomly selected from the Stockholm census register. All of the women were free of symptoms of heart disease and had not been hospitalized due to illness during the previous 5 years.

Compared with a random sample of 2500 women of the same age from the general population of Stockholm, the women in our study group were found to be similar in regard to educational level and such health behavior factors as smoking, exercise, and dietary habits (16). The study group could thus be regarded as representative of healthy women aged 30-65 years in the normal Swedish population.

The study was approved by the Karolinska Ethical Committee, and all of the participants gave their informed consent.

\section{Procedure}

The baseline examination was carried out between 1991 and 1994. Before the participants visited the research clinic, they received a questionnaire about their sleep, work strain, and psychosocial and standard risk factors for cardiovascular disease. The participants completed the questionnaire at home and brought it to the research clinic. A research nurse reviewed the questionnaires with the participants. Anthropometric measures and full lipid and routine laboratory profiles were obtained. The procedure has been described in detail elsewhere (15).

\section{Assessment of sleep complaints}

Disturbed sleep and feelings of tiredness were measured by the short version of the Karolinska Sleep Questionnaire (KSQ) $(13,17)$ in both the baseline and follow-up examinations. The women were asked about mental fatigue, not feeling well-rested on awakening, disturbed or restless sleep, premature awakening, and difficulties falling asleep. The response alternatives were "never", "rarely", "some of the time", and "most of the time". A disturbed sleep index was computed by the simple addition of all of the item scores (range 0 to 15). A higher score indicated more frequently disturbed sleep. For some analyses we used a categorical variable, sleep quality, and the women who answered "most of the time" for at least one sleep question (ie, disturbed sleep, premature awakening and difficulties falling asleep) and for mental fatigue or not feeling well-rested on awakening were categorized as having clinical insomnia. The women who responded with "most of the time" for at least one sleep question, but who did not report difficulties with tiredness, were categorized as having disturbed sleep, and the rest of the women were categorized as not having sleep difficulties.

\section{Assessment of work strain}

The psychosocial examination included questions on exposure to chronic work strain and was only measured at baseline. Work strain was measured using the Swedish version of the demand-control questionnaire, which has been tested for consistency and reliability in the Swedish population (18). The questions regarding demands concerned both qualitative and quantitative demands, whereas the question measuring job control covered skill discretion and authority over decision. All of the questions had four response categories (from never to always), and the scale scores were obtained by means of the simple addition of all of the item scores per scale. For the analyses, demands and control were dichotomized at the median. Then, following Karasek's model, we formed two groups. The women who had high demands but low control formed a high work-strain group. The remaining women formed a low work-strain group.

\section{Covariates}

Age at examination was derived from the 10-digit personal identification number of the census register. The 
participants were divided according to smoking habits into those who had never smoked, previous smokers, and current smokers. Physical exercise was assessed according to criteria of the World Health Organization (19) and was categorized as participation in heavy exercise, participation in mild exercise, or sedentary lifestyle. The participants' blood pressure, heart rate, weight, and height were measured by a research nurse during the clinical examination. Body mass index (BMI) was calculated as weight $(\mathrm{kg}) /$ height $\left(\mathrm{m}^{2}\right)$. The waist-to-hip ratio was calculated as the waist measurement $(\mathrm{cm})$ divided by the hip measurement $(\mathrm{cm})$. For the biochemical assessment, fasting venous blood samples were drawn at 0800. Total cholesterol was determined with standardized enzymatic methods. Fasting serum glucose was analyzed with the use of the GOD-PAP method.

\section{Nine-year follow-up}

Between April 2002 and August 2003 all of the control women were reexamined at the original research site. Because of the completeness of the Stockholm Census Registry and the individualized personal identification numbers, we were able to trace all of the women, even those few women who had moved to other countries. If a woman had died during the follow-up, time and cause of death were ascertained from her death certificate and hospital records. All of the women alive were contacted by letter. The short version of the baseline questionnaire, including the KSQ and questions about sick leave and hospitalization during the last 9 years, was included with additional questions on retirement (reason for and age at retirement). Most of the women contacted us spontaneously for an appointment; the remaining women received a second and third letter. Of the original 300 women, 264 women came to the research clinic, where the questionnaires were reviewed with them, and the questions with missing responses were completed. Weight, height, and waist-to-hip ratio were measured with the participant in a standing position without shoes. Blood pressure at rest was measured in the supine position after 5 minutes of rest. A medical history was obtained, including a complete history of medication, and a standardized medical examination was performed by a physician.

Thirteen women answered the questionnaire, but did not come to the research clinic. A few women who would not answer the full questionnaire agreed to answer the questions about their retirement $(\mathrm{N}=8)$. Three women who were, due to sickness, unable to come to the baseline examination answered the questionnaire and were visited and examined in their homes. One woman did not answer any questions. Eleven women died during the follow-up period; their death certificates and hospital charts were scrutinized.

\section{Employment, sick leave and retirement at follow-up}

The women were divided into the following three groups according to labor market status: employed $(\mathrm{N}=83)$, chronically ill $(\mathrm{N}=61)$ and retired $(\mathrm{N}=142)$. "Employed" was defined as working full-time, half-time, or less than half-time. Those who were on partial retirement were defined as employed. The two housewives were included in the employed group. The women who were on full or partial sick leave or who had been pensioned due to illness were defined as ill. Three of the unemployed women were classified as ill on the basis of their medical history. The women who had reached the retirement age of 65 years or who had been offered early retirement by their employers and those who retired because of their "own choice" (ie, without financial compensation) were defined as retired.

\section{Medical diagnosis in the follow-up}

Medical diagnoses for hospitalization or long-term sick leave from work over the 9 years of follow-up were confirmed from a scrutiny of the participants' medical records. If a woman had suffered from several diseases, the most dominant was considered to be the main diagnosis. The women were assigned to one of five of the following mutually exclusive diagnostic groups: (i) cancer ( $\mathrm{N}=26$ ); (ii) cardiovascular or cerebrovascular disease $(\mathrm{N}=25)$ or diabetes $(\mathrm{N}=2)$; (iii) musculoskeletal disease ( $\mathrm{N}=29)$; (iv) depression, burnout, or other psychiatric diseases $(\mathrm{N}=30)$; and $(\mathrm{v})$ other diagnoses, including infection $(\mathrm{N}=28)$ or benign gynecological, gastrointestinal, or other illnesses $(\mathrm{N}=10)$. In the analyses of the effects of sleep and stress on cardiocerebrovascular disease, we included the women who died as a result of cardiocerebrovascular disease $(\mathrm{N}=5)$ in the cardiocerebrovascular group.

Three groups were formed for the analyses. One group consisted of women with cardiocerebrovascular disease or diabetes $(\mathrm{N}=27)$. Another group contained all of the women who developed or died because of a disease other than cardiocerebrovascular disease or diabetes $(\mathrm{N}=123)$. Women who had not been on longterm sick leave or hospitalized during the last 9 years were defined as being healthy $(\mathrm{N}=140)$ and formed the third group.

\section{Statistical analysis}

Continuous data have been presented as means and standard deviations of the means, whereas categorical data have been shown as percentages. The differences between the three healthy groups with respect to the continuous variables were tested with an analysis of variance (ANOVA). The categorical data were compared 
using the $\chi^{2}$ test. One-way (group) ANOVA were used to evaluate the differences in the disturbed sleep index and age among the groups. To compare the disturbed sleep index at baseline and the follow-up, paired t-tests were carried out. The analyses for change were carried out using the data of the women who answered the sleep questionnaire both at baseline and at the followup. Multiple logistic regression analyses were used for calculating the independent relative risk of becoming ill in the different risk groups (ie, poor sleep, high work strain). In these analyses, all those ill (cardiocerebrovascular disease, diabetes, or other illness) were compared with the reference group of healthy persons. In the nonmultiple-adjusted analyses, we adjusted for age. In the multiple-adjusted analyses, we adjusted for age, glucose level, systolic blood pressure, cholesterol level, waist-to-hip ratio, exercise habits, smoking, and education. SAS 8.01 (SAS Inc, Gary, NC, USA) was used for the analyses.

\section{Results}

At baseline, all but one ( $\mathrm{N}=299)$ woman answered the questions about sleep. After an average of 9.2 (range 8.3-12.1) years of follow-up, 260 women answered the sleep questions again. The overall index did not change during the follow-up (rating 6.9 at baseline versus 7.2 at follow-up, $\mathrm{P}=0.18$ ).

Eleven women died during the follow-up. Five deaths were due to cardiocerebrovascular diseases, five were the result of cancer, and one woman committed suicide. Twenty-two women were hospitalized for cardiocerebrovascular disease or diabetes.

At the follow-up, we obtained information on the health status of 290 of the 300 women. Of these, 140 were found to be healthy [ie, had not been hospitalized or had not been on long-term sick leave ( $>28$ days) from work during the follow-up period]. Of the 27 women diagnosed with cardiocerebrovascular disease or diabetes, 19 had coronary disease, 6 patients had cerebrovascular disease, and 2 had diabetes. Five of these women had died because of cardiocerebrovascular disease. Complete medical reports, including in- and outpatient care, and death certificates with codes of the International Classification of Diseases (ICD), 10th revision, were available for 16 of the 27 women with cardiocerebrovascular disease (I10-I15 hypertensive diseases, I20-I25 ischemic heart diseases, I60-I69 cerebrovascular diseases) or diabetes (E10-E14). Partial medical reports, of in-patient care with ICD codes, were available for 11 women. Altogether 123 women had been diagnosed as having an illness other than cardiocerebrovascular disease or diabetes.
Table 1 shows a comparison of the standard risk factors for cardiovascular disease, the disturbed sleep index, and work strain at baseline between the women who developed cardiocerebrovascular disease or diabetes, those with another illness, and the healthy women. Major differences between the three groups were found for cholesterol level, exercise habits, and work strain. Cholesterol was highest, a sedentary lifestyle the most common, and work strain the most pronounced among the women who contracted cardiocerebrovascular disease or diabetes during the follow-up. We did not find any statistically significant differences between the groups with respect to age, BMI, waist-to-hip ratio, heart rate, systolic blood pressure, glucose level, smoking, or sleep quality (treated as a categorical variable).

Table 2 shows the disturbed sleep index as assessed at baseline and at the 9-year follow-up according to health status, employment status, and work strain. The women who developed cardiocerebrovascular disease or diabetes during the follow-up and those who developed another illness more frequently reported disturbed sleep at baseline than the women who remained healthy. This difference reached borderline statistical significance $(\mathrm{P}=0.07)$. At the follow-up, both of the groups with illnesses more frequently reported disturbed sleep than the women who had remained healthy $(\mathrm{P}=0.002)$. The Bonferroni t-tests yielded significant differences in sleep quality between the healthy women and the women who developed cardiocerebrovascular disease or diabetes. Those with cardiocerebrovascular disease or diabetes at the follow-up had a significantly higher disturbed sleep index than the two other groups. There was no age difference between the groups.

Sleep disturbances at baseline did not differ between the younger $(<58$ years, $\mathrm{N}=141)$ and older $(\geq 58$ years, $\mathrm{N}=151$ ) women, 7.2 (SD 3.1) versus 6.6 (SD 3.3) $(\mathrm{P}=0.12)$. However, during the follow-up, the younger women's disturbed sleep index increased over time $(\mathrm{P}<0.01)$ to a higher level than that of the older women, 7.9 (SD 3.1) versus 6.4 (SD $3.0(\mathrm{P}<0.001)$. The older women's disturbed sleep index did not change over time.

In a further analysis, we combined the women with cardiocerebrovascular disease or diabetes $(\mathrm{N}=27)$ and those with another illness $(\mathrm{N}=123)$ into one group: sick women $(\mathrm{N}=150)$. We compared these sick women with the women who remained healthy $(\mathrm{N}=140)$. We found that the women who became ill during the follow-up had a statistically significantly worse sleep quality at baseline and at the follow-up than the women who remained healthy [disturbed sleep index at baseline: 7.3 (SD 3.0) versus 6.5 (SD 3.2) $(\mathrm{P}=0.04)$; disturbed sleep index at follow-up: 7.8 (SD 3.1) versus 6.6 (SD $3.0(\mathrm{P}=0.004)$ ].

In the comparison of the sick women $(\mathrm{N}=145)$ with the healthy women $(\mathrm{N}=137)$, the logistic regression 
Table 1. Baseline characteristics of the women who, by the time of the follow-up, had contracted cardiocerebrovascular disease or diabetes $(\mathrm{N}=27)$, those who developed diseases other than cardiocerebrovascular disease or diabetes $(\mathrm{N}=123)$ and those who remained healthy $(\mathrm{N}=140)$. $(\mathrm{BMI}=$ body mass index $)$

\begin{tabular}{|c|c|c|c|c|c|c|c|c|c|c|c|c|c|}
\hline \multirow[t]{2}{*}{ Characteristic } & \multicolumn{4}{|c|}{$\begin{array}{l}\text { Women who contracted cardio- } \\
\text { celebrovascular disease or diabetes }\end{array}$} & \multicolumn{4}{|c|}{$\begin{array}{c}\text { Women who contracted } \\
\text { other disease }\end{array}$} & \multicolumn{4}{|c|}{$\begin{array}{l}\text { Women who } \\
\text { remained healthy }\end{array}$} & \multirow[t]{2}{*}{$\begin{array}{c}\text { P- } \\
\text { value }\end{array}$} \\
\hline & Mean & $\mathrm{SD}$ & $\mathrm{N}$ & $\%$ & Mean & SD & $\mathrm{N}$ & $\%$ & Mean & $\mathrm{SD}$ & $\mathrm{N}$ & $\%$ & \\
\hline Age (years) & 58.1 & 6.3 & . & . & 55.4 & 7.0 & . & . & 57.1 & 7.3 & . & . & $0.06^{a}$ \\
\hline BMI $\left(\mathrm{kg} / \mathrm{m}^{2}\right)$ & 26.1 & 4.6 & . & . & 26.1 & 5.4 & . & . & 24.9 & 3.9 & . & - & $0.10^{\mathrm{a}}$ \\
\hline Waist-to-hip ratio (cm) & 0.83 & 0.08 & $\cdot$ & . & 0.80 & 0.09 & . & . & 0.79 & 0.09 & . & . & $0.16^{\mathrm{a}}$ \\
\hline Heart rate (beats/min) & 66.9 & 9.3 & . & . & 68.2 & 11.6 & . & . & 69.3 & 11.0 & . & . & $0.51^{\mathrm{a}}$ \\
\hline Systolic blood pressure & 120.7 & 13.4 & . & . & 119.5 & 16.0 & . & . & 121.9 & 17.9 & . & . & $0.52^{a}$ \\
\hline Cholesterol level (mmol/l) & 6.5 & 1.3 & . & . & 5.8 & 0.9 & . & . & 6.2 & 1.1 & . & . & $<0.001^{a}$ \\
\hline Glucose level (mmol/l) & 5.0 & 0.7 & $\cdot$ & . & 5.0 & 0.5 & . & . & 4.9 & 0.5 & - & . & $0.53^{a}$ \\
\hline Smoking habits & & & & & & & & & & & & & $0.99^{b}$ \\
\hline Never & . & . & 12 & 44.4 & . & . & 57 & 46.3 & . & . & 65 & 46.4 & . \\
\hline Previous & . & . & 7 & 25.9 & . & . & 29 & 23.6 & . & . & 36 & 25.7 & . \\
\hline Current & - & - & 8 & 29.6 & . & - & 37 & 30.1 & . & . & 39 & 27.9 & - \\
\hline Exercise habits & & & & & & & & & & & & & $<0.01^{b}$ \\
\hline Sedentary & . & . & 11 & 42.3 & . & - & 24 & 19.7 & . & . & 16 & 11.5 & . \\
\hline Mild exercise & . & . & 13 & 50.0 & . & . & 84 & 68.9 & . & . & 109 & 78.4 & . \\
\hline Heavy exercise & - & . & 2 & 7.7 & . & - & 14 & 11.5 & . & . & 14 & 10.1 & . \\
\hline Sleep quality c & & & & & & & & & & & & & $0.82^{b}$ \\
\hline Good quality & . & . & 20 & 76.9 & . & - & 102 & 82.9 & . & . & 114 & 81.4 & . \\
\hline Disturbed sleep & . & . & 3 & 11.5 & . & - & 14 & 11.4 & . & . & 18 & 12.9 & . \\
\hline Insomnia & $\cdot$ & . & 3 & 11.5 & . & - & 7 & 5.7 & . & . & 8 & 5.7 & \\
\hline Work strain & & & & & & & & & & & & & $0.02^{b}$ \\
\hline Low & . & . & 19 & 86.4 & . & . & 65 & 67.1 & . & . & 83 & 82.2 & . \\
\hline High & . & . & 3 & 13.6 & . & . & 32 & 33.0 & . & . & 18 & 17.8 & . \\
\hline
\end{tabular}

a ANOVA.

b Chi-square.

c Good quality: no sleep problems most of the time; distrubed sleep: at least one sleep problem most of the time but no tiredness; insomnia: at least one sleep problem most of the time and also tiredness.

Table 2. Disturbed sleep index at baseline and after 9 years of follow-up in relation to cardiocerebrovascular health, employment, and work strain. (CCVD = cardocerebrovascular disease)

\begin{tabular}{|c|c|c|c|c|c|c|c|c|c|c|}
\hline & \multirow[t]{2}{*}{$\mathrm{N}$} & \multicolumn{3}{|c|}{ Baseline index ${ }^{a}$} & \multirow[t]{2}{*}{ N } & \multicolumn{3}{|c|}{ Follow-up index a } & \multicolumn{2}{|c|}{ P-value ${ }^{b}$} \\
\hline & & Mean & SD & $\mathrm{P}$-value ${ }^{\mathrm{C}}$ & & Mean & SD & P-value ${ }^{c}$ & Change & $N^{d}$ \\
\hline \multicolumn{11}{|c|}{ Cardiocerebrovascular health at follow-up } \\
\hline $\begin{array}{l}\text { Healthy (mean } 57.1, \text { SD } 6.3) \\
\text { Other illness (mean 55.4, SD 7.0) } \\
\text { CCVD (mean 55.4, SD 7.0) } \\
\mathrm{P}_{\text {age }}{ }^{b}=0.59\end{array}$ & $\begin{array}{r}140 \\
123 \\
27\end{array}$ & $\begin{array}{l}6.5 \\
7.2 \\
7.9\end{array}$ & $\begin{array}{l}3.2 \\
3.1 \\
2.9\end{array}$ & 0.07 & $\begin{array}{r}124 \\
104 \\
19\end{array}$ & $\begin{array}{l}6.6 \\
7.5 \\
9.1\end{array}$ & $\begin{array}{l}3.0 \\
3.3 \\
1.7\end{array}$ & 0.002 & $\begin{array}{r}0.92 \\
0.28 \\
<0.01\end{array}$ & $\begin{array}{r}122 \\
102 \\
18\end{array}$ \\
\hline \multicolumn{11}{|l|}{ Employment status at follow-up e } \\
\hline $\begin{array}{l}\text { Employed (mean 49.9, SD 5.6) } \\
\text { Retired (mean 60.9, SD 3.9) } \\
\text { Sick leave (mean 53.8, SD 6.9) } \\
P_{\text {age }}{ }^{b}<0.0001\end{array}$ & $\begin{array}{r}83 \\
142 \\
61\end{array}$ & $\begin{array}{l}6.6 \\
6.7 \\
7.9\end{array}$ & $\begin{array}{l}2.9 \\
3.3 \\
3.3\end{array}$ & 0.03 & $\begin{array}{r}77 \\
122 \\
48\end{array}$ & $\begin{array}{l}7.3 \\
6.5 \\
8.7\end{array}$ & $\begin{array}{l}2.8 \\
3.0 \\
3.4\end{array}$ & $<0.001$ & $\begin{array}{l}0.03 \\
0.45 \\
0.21\end{array}$ & $\begin{array}{r}75 \\
121 \\
46\end{array}$ \\
\hline \multicolumn{11}{|l|}{ Work strain at baseline ${ }^{e}$} \\
\hline $\begin{array}{l}\text { Low (mean 54.6, SD 6.9) } \\
\text { High (mean 54.9, SD 6.8) } \\
P_{\text {age }}{ }^{b}=0.80\end{array}$ & $\begin{array}{r}168 \\
54\end{array}$ & $\begin{array}{l}6.3 \\
8.1\end{array}$ & $\begin{array}{l}3.1 \\
3.1\end{array}$ & $<0.001$ & $\begin{array}{r}147 \\
47\end{array}$ & $\begin{array}{l}7.1 \\
7.7\end{array}$ & $\begin{array}{l}3.2 \\
3.0\end{array}$ & 0.24 & $\begin{array}{r}<00.01 \\
0.11\end{array}$ & $\begin{array}{r}145 \\
45\end{array}$ \\
\hline
\end{tabular}

a Scored 0-15, higher score means a more disturbed sleep.

c $\mathrm{P}$-value for the analysis of variance.

${ }^{b} \mathrm{P}$-value for paired t-test.

a Number of individuals in the entire analysis.

${ }^{e}$ The mean age and standard deviations are given in parentheses after the categories. 
analysis yielded a $10 \%$ increase in the risk of developing any disease for each increment increase in the disturbed sleep index [odds ratio (OR) 1.1, 95\% confidence interval (95\% CI) 1.0-1.2]. After multivariate adjustment for age, glucose level, systolic blood pressure, cholesterol level, waist-to-hip ratio, exercise habits, smoking, and education, the increased risk for developing any disease remained significant (OR 1.1, 95\% CI 1.0-1.2). When we compared the women who developed cardiocerebrovascular disease or diabetes with all of the other women, we did not find an increased risk. However, when we compared the women with cardiocerebrovascular disease or diabetes with the healthy women only, we found a $15 \%$ increased risk for developing cardiocerebrovascular disease or diabetes (OR 1.15, 95\% CI 1.0-1.3).

Employment status was associated with the disturbed sleep index. The Bonferroni t-tests yielded significant differences between the women on sick leave on one hand and the women who were working or retired on the other, in regard to the disturbed sleep index at both the baseline $(\mathrm{P}=0.03)$ and the follow-up $(\mathrm{P}<0.001)$. No difference was found between the working and retired women. The disturbed sleep index of the retired women and the women on sick leave did not change during the follow-up period, but the working women had a significantly higher disturbed sleep index (table 2).

Work strain was associated with the disturbed sleep index at baseline $(\mathrm{P}<0.001)$, but not at the follow-up. At baseline the women with high work strain more frequently had disturbed sleep than the women with low work strain. During the follow-up, the disturbed sleep index of the women with low work strain increased to the same level as that of the women with high work strain.

The logistic regression yielded a nearly doubled risk for becoming sick (cardiocerebrovascular disease or diabetes or another illness) for the high work-strain group $(\mathrm{N}=53)$ when it was compared with the low work-strain group ( $\mathrm{N}=167)$ (OR 1.9, 95\% CI 1.0-3.7). After multiple adjustment for age, glucose level, systolic blood pressure, cholesterol level, waist-to-hip ratio, exercise habits, smoking habits, and education, the risk increased somewhat (OR 2.3, 95\% CI 1.1-4.7). No increased risk for developing cardiocerebrovascular disease was found.

\section{Discussion}

Of the originally healthy control women participating in The Stockholm Female Coronary Risk Study, 11 women $(0.4 \%)$ died during the 9 years of follow-up. Only about half of the initially healthy middle-aged women remained healthy during the 9 years of follow-up, and half of the women had been or were hospitalized or were on long-term sick leave. The most serious diseases (malignant tumors and cardiocerebrovascular diseases) were about equally common both as a cause of death and as a cause of disability and hospitalization.

Among the clinical risk factors, we found only two that were associated with cardiocerebrovascular disease or diabetes in a long-term perspective. Compared with the women who developed another disease, those who developed a cardiocerebrovascular disease or diabetes or who remained healthy had an increased cholesterol level. The waist-to-hip ratio was slightly higher among the women who contracted cardiocerebrovascular disease or diabetes. Significant differences were found for exercise habits and work strain. The women who stated at baseline that they exercised frequently developed cardiocerebrovascular disease or diabetes less often. Our findings confirm that a sedentary lifestyle has a negative influence on cardiovascular health $(20,21)$ even if studies of women have produced mixed results (22). Work strain was not associated with a specific risk for cardiocerebrovascular disease, but it was associated with an overall increased risk of becoming ill. The disturbed sleep index indicated that the women who developed cardiocerebrovascular disease or diabetes or another disease experienced more disturbed sleep than the women who remained healthy-already at baseline. Perhaps a higher disturbed sleep index among the women with future illness is an early sign of, and a reason for, an ongoing redevelopment of ill health, even though the clinical presentation has not yet been affected. One might suggest that poor sleep depends on a sedentary lifestyle, as it is known that physical activity improves sleep quality and is often recommended to patients with sleep difficulties $(23,24)$. In our study, $11 \%$ of the women with a sedentary lifestyle suffered from insomnia, whereas only $3 \%$ of the women who frequently exercised did so.

The large difference between the insomnia variable and the continuous disturbed sleep index in relation to the health outcomes was unexpected. The results showed that only a few of the participants in our sample fulfilled the insomnia criteria, whereas the disturbed sleep index showed a large interindividual variation. It is obvious that the disturbed sleep index was able to pick up moderate sleep disturbances [eg, occasional problems that do not occur (almost) every night]. Hence, the disturbed sleep index seems to have been more statistically sensitive than the categorical insomnia variable. A practical implication of this assumption is that moderate sleep disturbances may be enough to cause negative health consequences; however, this speculation needs further study.

We found that disturbed sleep was related to an increased risk for developing cardiocerebrovascular disease or diabetes. This risk did not remain after control 
for lifestyle, education, and physiological risk factors. However, disturbed sleep was related to an increased risk of other illness. These increased risk levels remained after multivariate adjustment. This finding is in line with the results of Nilsson et al (3). They found that insomnia, adjusted for age and other risk factors, was a risk marker for total and "other" mortality among women, but not for cardiovascular disease. However, other longitudinal studies have shown that subjective sleep complaints predict death from acute myocardial infarction or coronary heart disease (10). In a previous prospective follow-up study of female patients, we reported a more than doubled risk for a recurrent cardiac event (12). Furthermore, we found that snoring contributes to the progression of the atherosclerotic process (25), providing a mechanism for the effect.

Employment status was associated with disturbed sleep both at baseline and at the follow-up. We found a significant difference in the disturbed sleep index between the employed or retired women and the women on sick leave both at baseline and at the follow-up. Disturbed sleep at baseline seemed to be an early sign of ill health, independent of the cause of the illness. This finding is in accordance to the results of previous work. Thus Dew et al (26) found that older persons with objectively measured worse sleep quality at baseline scored higher on a measure of cumulative medical burden in a 1-year follow-up. In a 4- to 19-year follow-up of healthy older adults, they further found that poor sleep (polysomnographically recorded) predicted all-cause mortality (27). Independent studies (6-8) have shown that mortality rates are higher among adults who habitually sleep less than 7 hours or more than 8 hours a night than they are for normal sleepers (7-8 hours of sleep) after control for age, health, and other risk factors. The poorer sleep at the follow-up among the women on sick leave in our study was not surprising since health and sleep quality are strongly associated (28). Poor sleep is not only a predictor of poor health, it is also a symptom of a medical disorder.

Work strain was also associated with disturbed sleep at baseline, but it did not predict cardiocerebrovascular disease and was not associated with disturbed sleep at the follow-up. Insomnia is usually considered a result of long-term stress (29), and sleep is usually closely related to stress $(13,14,30,31)$. High job strain can cause problems with unwinding (8) and, therefore, cause problems with falling asleep. However, work strain at baseline in our study did not predict poor sleep 9 years later. This finding is hardly surprising, as about half of the women were no longer employed. It seems that high work strain affects sleep only as long as it is ongoing and that the poor effects of work strain on sleep disappear soon after the strain has been removed. Another possibility is that work strain decreased because of improved work conditions during the time between the baseline and the follow-up measurements.

It should be considered whether a questionnaire is a reliable and valid method for measuring sleep quality. However, the questionnaire is the most frequently used method for estimating sleep disturbances, particularly in epidemiologic studies. A traditional one-night polysomnographic sleep recording is costly, and it does not necessarily reflect trait-like sleep quality. Therefore, it is difficult to validate the subjective instruments against physiological measures. However, studies on objective and subjective sleep quality have shown that sleep latency, sleep length, and the number of awakenings often differ according to the method used (32). On the other hand, recent analyses of the KSQ show that the correspondence between the KSQ and a sleep diary, validated against electroencephalographic data, is strong (33). Thus the sleep questionnaire was a strong predictor of the frequency of poor sleep in the diary.

It may also be that the disturbed sleep index reflects other medical disorders, in particular those related to mental health. It is well known that poor sleep is a core symptom in depression and anxiety disorders. In addition, the disturbed sleep index may also have captured clinical sleep disorders such as snoring and sleep apnea. Furthermore, the study population included only women under the age of 65 years; therefore it can be difficult to draw conclusions concerning older women. Women suffer from sleep difficulties far more often than men (4, 34) but are often underrepresented in studies concerning sleep and coronary heart disease.

A general concern of this paper was the relatively small number of women included in the different diagnostic groups. Hence, the findings need to be replicated with the use of a larger sample.

We conclude that general and cardiovascular health are important factors for middle-aged women's subjectively rated sleep quality and suggest that poor sleep can, to a certain degree, be an early sign of subclinical cardiac and cerebrovascular disease.

\section{Acknowledgments}

This work was funded by grant HL 45785 from the United States National Institutes of Health, the Swedish Bank Tercentenary Fund, the Swedish Medical Research Council, and the Swedish Council for Work Life Research and the Swedish Labor Market Insurance Company (twice).

Special thanks go to Evelyn Lewandrowski for the excellent coordination of the follow-up examination of the women. 


\section{References}

1. Elmasry A, Janson C, Lindberg E, Gislason T, Tageldin MA, Boman G. The role of habitual snoring and obesity in the development of diabetes: a 10-year follow-up study in a male population. J Intern Med. 2000;248:13-20.

2. Mallon L, Hetta J. A survey of sleep habits and sleeping difficulties in an elderly Swedish population. Ups J Med Sci. 1997;102(3):185-97.

3. Nilsson PM, Nilsson J-Å, Hedblad B, Berglund G. Sleep disturbance in association with elevated pulse rate for prediction of mortality - consequences of mental strain? J Intern Med. 2001;250:1-9.

4. Ohayon MM. Epidemiology of insomnia: what we know and what we still need to learn. Sleep Med Rev. 2002;6:97-111.

5. Ford DE, Kamerow DB. Epidemiologic study of sleep disturbances and psychiatric disorder: a opportunity for prevention. JAMA. 1989;262 (11):1479-84.

6. Wingard DL, Berkman LF. Mortality risk associated with sleeping patterns among adults. Sleep. 1983;6:102-7.

7. Kripke DF, Simons RN, Garfinkel L, Hammond EC. Short and long sleep and sleeping pills: is increased mortality associated. Arch Gen Psychiatry. 1979;36:103-16.

8. Kripke DF, Garfinkel L, Wingard DL, Klauber MR, Marler MR. Mortality associated with sleep duration and insomnia. Arch Gen Psychiatry. 2002;59(2):131-6.

9. Schwartz SW, Coroni-Huntley J, Cole SR, Hays JC, Blazer DG, Schockern DD. Are sleep complaints an independent risk factor for myocardial infarction? Ann Epidemiol. 1998;8(6):384-92.

10. Schwartz K, Anderson MW, Cole SR, Cornoni-Huntley J, Hays JC, Blazer D. Insomnia and heart disease: a review of epidemiologic studies. J Psychosom Res. 1999;47(4):313-33.

11. Eaker ED, Pinsky J, Castelli WP. Myocardial infarction and coronary death among women: psychosocial predictors from a 20-years follow-up of women in the Framingham Study. Am J Epidemiol. 1992;135:854-64.

12. Leineweber C, Kecklund G, Janszky I, Akerstedt T, OrthGomer K. Poor sleep increases the prospective risk for recurrent events in middle-aged women with coronary disease: The Stockholm Female Coronary Risk Study. J Psychosom Res. 2003;54:121-7.

13. Åkerstedt T, Knutsson A, Westerholm P, Theorell T, Alfredsson L, Kecklund G. Sleep disturbances, work stress and work hours: a cross-sectional study. J Psychosom Res. 2002;53(3):741-8.

14. Kecklund G, Åkerstedt T. Apprehension of the subsequent working day is associated with a low amount of slow wave sleep. Biol Psychol. 2004;66:169-76.

15. Orth-Gomér K, Mittleman M, Schenck-Gustafsson K, Wamala S, Eriksson M, Belkic K, et al. Lipoprotein (a) as a determinant of coronary heart disease in young women. Circulation. 1997;95:329-34.

16. Romelsjö A, Hasin D, Hilton M, Foström G, Diderichsen F, Haglund B, et al. The relationship between stressful working conditions and high alcohol consumption and severe alcohol problems in an urban general population. Br J Addict. 1992;87:1173-83.
17. Kecklund G, Akerstedt T. The psychometric properties of the Karolinska Sleep Questionnaire. J Sleep Res. 1992;6:221-9.

18. Karasek R, Baker D, Marxer F, Ahlbom A, Theorell T. Job decision latitude, job demands and cardiovascular disease: a prospective study of Swedish men. Am J Public Health. 1981;71:694-705.

19. Baecke JA, Burema J, Frijters JE. A short questionnaire for the measurement of habitual physical activity in epidemiological studies. Am J Clin Nutr. 1982;36(5):936-42.

20. Smith G, Shipley M, Batty G, Morris J, Marmot M. Physical activity and cause-specific mortality in the Whitehall study. Public Health. 2000;114(5):308-15.

21. Andersen LB. Physical activity and physical fitness as protection against premature disease or death. Scand J Med Sci Sports. 1995; 5 (6):318-28.

22. Barrett-Connor E. Sex differences in coronary heart disease: why are women so superior?: the 1995 Ancel Keys Lecture. Circulation. 1997;95(1):252-64.

23. Montgomery P, Dennis J. Physical exercise for sleep problems in adults aged 60+. Cochrane Database Syst Rev 2002;4(CD03404).

24. Dement WC. The promise of sleep. New York (NY): Delacorte Press; 1999.

25. Leineweber C, Kecklund G, Janszky I, Akerstedt T, Orth-Gomér K. Snoring and progression of coronary artery disease: The Stockholm Female Coronary Angiography Study. Sleep. 2004; 27 (7):1344-9.

26. Dew MA, Reynolds III CF, Monk TH, Buysse DJ, Hoch CC, Jennings R, et al. Psychosocial correlates and sequelae of electroencephalographic sleep in healthy elders. J Gerontol. 1994;49(1):8-18.

27. Dew MA, Hoch CC, Buysse DJ, Monk TH, Begley AE, Houck PR, et al. Healthy older adults' sleep predicts all-cause mortality at 4 to 19 years of follow-up. Psychosom Med. 2003;65(1):63-73.

28. Fabsitz RR, Sholinsky P, Goldberg JD. Correlates of sleep problems among men: The Vietnam Era Twin Registry. J Sleep Res. 1997;6:50-6.

29. Kryger MH, Roth T, Dement WC, editors. Principles and practice of sleep medicine. 2nd ed. Philadelphia (PA): WB Saunders Company; 1994.

30. Urponen H, Vuori I, Hasan J, Partinen M. Self-evaluations of factors promoting and disturbing sleep: an epidemiological survey in Finland. Soc Sci Med. 1988;26(4):443-50.

31. Marquié JC, Foret J, Queinnec Y. Effects of age, working hours, and job content on sleep: a pilot study. Exp Aging Res. 1999;25:421-7.

32. Bonnet MH, Arand DL. Hyperarousal and insomnia. Sleep Med Rev. 1997;1(2):97-108.

33. Axelsson J, Kecklund G, Åkerstedt T, Ekstedt M, Menega J. A comparison of the Karolinska Sleep Questionnaire and the Karolinska Sleep Diary: a methodological study [abstract]. J Sleep Res. 2002;11 suppl 1:8.

34. Ohayon MM, Caulet M, Arbus L, Billard M, Coquerel A, Guieu $\mathrm{JD}$, et al. Are prescribed medications effective in the treatment of insomnia complaints? J Psychosom Res. 1999;47:359-68.

Received for publication: 18 November 2005 